\title{
Single-Crystal to Single-Crystal Phase Transitions of Commensurately Modulated Sodium Saccharinate 1.875-Hydrate
}

Toms Rekis, Achim M. Schaller, Surya Rohith Kotla, Andreas Schönleber, Leila Noohinejad, Martin Tolkiehn, Carsten Paulmann, Sander van Smaalen* smash@uni-bayreuth.de

Supporting information

\begin{tabular}{|c|c|c|c|c|c|c|c|c|}
\hline Phase & I & I & I & I & I & I & I & I \\
\hline$T / \mathrm{K}$ & $298^{2 \mathrm{nd}}$ & 298 & 270 & 250 & 210 & 170 & 140 & 130 \\
\hline \multicolumn{9}{|l|}{ Crystal data } \\
\hline Formula & \multicolumn{8}{|c|}{$\mathrm{Na}\left(\mathrm{C}_{7} \mathrm{H}_{4} \mathrm{NO}_{3} \mathrm{~S}\right) \cdot{ }^{15} / 8 \mathrm{H}_{2} \mathrm{O}$} \\
\hline Formula w. & \multicolumn{8}{|c|}{238.94} \\
\hline Crystal system & \multicolumn{8}{|c|}{ Monoclinic } \\
\hline Space group & \multicolumn{8}{|c|}{$C 2 / c\left(0, \sigma_{2}, 0\right) s 0$} \\
\hline $\mathbf{q}, t_{0}$ & \multicolumn{8}{|c|}{$(0,3 / 4,0), 0$} \\
\hline a/ $\AA$ & $18.74881(2)$ & $18.74705(8)$ & $18.72290(9)$ & $18.70776(6)$ & $18.67047(5)$ & $18.62995(7)$ & $18.60326(8)$ & $18.59400(5)$ \\
\hline$b / \AA$ & $7.15262(4)$ & $7.15189(8)$ & $7.14263(9)$ & $7.13587(8)$ & $7.12303(9)$ & $7.11507(8)$ & $7.10726(6)$ & $7.10397(7)$ \\
\hline$c / \AA$ & $29.18116(4)$ & $29.18066(11)$ & $29.16831(9)$ & $29.15543(10)$ & $29.12916(10)$ & $29.12876(8)$ & $29.11976(7)$ & $29.11214(5)$ \\
\hline$\beta /^{\circ}$ & $93.77247(5)$ & $93.77227(9)$ & $93.75256(7)$ & $93.73321(6)$ & $93.66445(5)$ & $93.55680(7)$ & $93.49728(10)$ & $93.48267(6)$ \\
\hline$V / \AA^{3}$ & $3904.80(2)$ & $3903.97(4)$ & $3892.34(4)$ & $3883.88(4)$ & $3865.98(4)$ & $3853.68(4)$ & $3842.99(3)$ & $3838.36(3)$ \\
\hline$Z, Z^{\prime}$ & 16,2 & 16,2 & 16,2 & 16,2 & 16,2 & 16,2 & 16,2 & 16,2 \\
\hline$D_{x} / \mathrm{g} \mathrm{cm}^{-3}$ & 1.6001 & 1.6004 & 1.6052 & 1.6087 & 1.6141 & 1.6213 & 1.6258 & 1.6278 \\
\hline$\mu / \mathrm{mm}^{-1}$ & 0.143 & 0.143 & 0.144 & 0.144 & 0.144 & 0.145 & 0.145 & 0.146 \\
\hline Meas. refl. & 219920 & 219580 & 218918 & 218346 & 217545 & 216584 & 215953 & 216106 \\
\hline$[\sin (\theta) / \lambda]_{\max } / \AA^{-1}$ & \multicolumn{8}{|c|}{$\frac{1}{0.75}$} \\
\hline Unique refl. & 53543 & 53580 & 53419 & 53302 & 53016 & 52778 & 52571 & 52512 \\
\hline Obs. refl. & 23243 & 26775 & 27512 & 28963 & 30241 & 32872 & 34556 & 34771 \\
\hline$R_{\text {int }}($ obs. $)$ & 0.0368 & 0.0388 & 0.0389 & 0.0383 & 0.0401 & 0.0371 & 0.0354 & 0.0353 \\
\hline \multicolumn{9}{|l|}{ Refinement } \\
\hline Ref. method & \multicolumn{8}{|c|}{ Full-matrix least-squares on $F$} \\
\hline No. of param. & 1702 & 1702 & 1702 & 1702 & 1702 & 1702 & 1702 & 1702 \\
\hline$R_{1}$ (obs.) & 0.0548 & 0.0539 & 0.0536 & 0.0534 & 0.0518 & 0.0499 & 0.0475 & 0.0471 \\
\hline$w R$ (all) & 0.0567 & 0.0563 & 0.0559 & 0.0555 & 0.0544 & 0.0530 & 0.0517 & 0.0515 \\
\hline GoF (all) & 1.74 & 1.95 & 1.95 & 2.03 & 2.04 & 2.14 & 2.21 & 2.22 \\
\hline$R_{\mathrm{m}=0}$ (obs.) & 0.0472 & 0.0498 & 0.0469 & 0.0474 & 0.0458 & 0.0442 & 0.0426 & 0.0426 \\
\hline$R_{\mathrm{m}= \pm 1}$ (obs.) & 0.0521 & 0.0504 & 0.0510 & 0.0496 & 0.0486 & 0.0471 & 0.0450 & 0.0451 \\
\hline$R_{\mathrm{m}= \pm 2}$ (obs.) & 0.0583 & 0.0565 & 0.0561 & 0.0567 & 0.0545 & 0.0521 & 0.0494 & 0.0486 \\
\hline$R_{\mathrm{m}= \pm 3}$ (obs.) & 0.0575 & 0.0557 & 0.0570 & 0.0557 & 0.0545 & 0.0524 & 0.0491 & 0.0488 \\
\hline$R_{\mathrm{m}= \pm 4}$ (obs.) & 0.0727 & 0.0677 & 0.0670 & 0.0681 & 0.0640 & 0.0610 & 0.0573 & 0.0558 \\
\hline H-atom treatment & constr. & constr. & constr. & constr. & constr. & constr. & constr. & constr. \\
\hline Weighting sch. & \multicolumn{8}{|c|}{$\left.w=1 / \sigma^{2}(F)+0.0001 F^{2}\right)$} \\
\hline$\Delta \rho_{\max } /$ e $\AA^{-3}$ & 1.10 & 0.86 & 1.03 & 1.22 & 1.42 & 1.26 & 1.41 & 1.17 \\
\hline$\Delta \rho_{\min } /$ e $\AA^{-3}$ & -1.03 & -0.91 & -1.01 & -0.93 & -1.34 & -1.56 & -1.52 & -1.53 \\
\hline
\end{tabular}




\begin{tabular}{|c|c|c|c|c|c|c|c|c|c|}
\hline Phase & II & II & II & II & II & II & II & III & III \\
\hline$T / \mathrm{K}$ & 120 & 110 & 100 & 90 & 80 & 70 & 60 & 40 & 20 \\
\hline \multicolumn{10}{|l|}{ Crystal data } \\
\hline Formula & \multicolumn{9}{|c|}{$\mathrm{Na}\left(\mathrm{C}_{7} \mathrm{H}_{4} \mathrm{NO}_{3} \mathrm{~S}\right) \cdot{ }^{15} / 8 \mathrm{H}_{2} \mathrm{O}$} \\
\hline Formula w. & \multicolumn{9}{|c|}{238.94} \\
\hline Crystal system & \multicolumn{7}{|c|}{ Monoclinic } & \multicolumn{2}{|c|}{ Triclinic* } \\
\hline Space group & \multicolumn{7}{|c|}{$C 2 / c\left(0, \sigma_{2}, 0\right) s 0$} & \multicolumn{2}{|c|}{$C-1\left(\sigma_{1}, \sigma_{2}, \sigma_{3}\right) 0$} \\
\hline $\mathbf{q}, t_{0}$ & \multicolumn{7}{|c|}{$(0,3 / 4,0), 0$} & \multicolumn{2}{|c|}{$(0,3 / 4,0), 0$} \\
\hline$a / \AA$ & $18.60894(7)$ & $18.60517(11)$ & $18.60142(5)$ & $18.59790(4)$ & $18.59125(4)$ & $18.58801(6)$ & $18.58299(3)$ & $18.57290(10)$ & $18.57670(10)$ \\
\hline$c / \AA$ & $29.07549(9)$ & $29.07473(5)$ & $29.07316(10)$ & $29.07311(5)$ & $29.06112(9)$ & $29.06013(7)$ & $29.04898(6)$ & $29.02330(10)$ & $29.04080(10)$ \\
\hline$\beta /^{\circ}$ & $93.59123(8)$ & $93.58434(12)$ & $93.58574(7)$ & $93.58194(6)$ & $93.55830(5)$ & $93.56348(5)$ & 93.56293(4) & $93.51100(10)^{* *}$ & $93.53420(10)^{* * *}$ \\
\hline$V / \AA^{3}$ & $3831.92(3)$ & 3830.31 & $3828.72(3)$ & $3827.24(3)$ & $3822.18(3)$ & $3821.33(4)$ & $3817.88(3)$ & $3810.31(6)$ & $3815.71(6)$ \\
\hline$Z, Z^{\prime}$ & 16,2 & 16,2 & 16,2 & 16,2 & 16,2 & 16,2 & 16,2 & 16,4 & 16,4 \\
\hline$D_{x} / \mathrm{g} \mathrm{cm}^{-3}$ & 1.6305 & 1.6312 & 1.6319 & 1.6325 & 1.6346 & 1.635 & 1.6365 & 1.6397 & 1.6374 \\
\hline$\mu / \mathrm{mm}^{-1}$ & 0.146 & 0.146 & 0.146 & 0.146 & 0.146 & 0.146 & 0.146 & 0.147 & 0.146 \\
\hline Meas. refl. & 215736 & 215742 & 215354 & 215114 & 215267 & 215148 & 214969 & 214582 & 214563 \\
\hline$[\sin (\theta) / \lambda]_{\max } / \AA^{-1}$ & \multicolumn{9}{|c|}{0.75} \\
\hline Obs. refl. & 34304 & 34814 & 34863 & 35261 & 35241 & 35507 & 36098 & 34261 & 34027 \\
\hline$R_{\text {int }}$ & 0.0360 & 0.0363 & 0.0370 & 0.0367 & 0.0403 & 0.0399 & 0.0367 & 0.0657 & 0.0793 \\
\hline \multicolumn{10}{|l|}{ Refinement } \\
\hline Ref. method & \multicolumn{9}{|c|}{ Full-matrix least-squares on $F$} \\
\hline No. of param. & 1702 & 1702 & 1702 & 1702 & 1702 & 1702 & 1702 & 2045 & 2045 \\
\hline$R_{1}$ (obs.) & 0.0492 & 0.0496 & 0.0491 & 0.0486 & 0.0489 & 0.0492 & 0.0482 & 0.0582 & 0.0665 \\
\hline$w R$ (all) & 0.0532 & 0.0535 & 0.0533 & 0.0534 & 0.0536 & 0.0537 & 0.0535 & 0.0610 & 0.0695 \\
\hline GoF (all) & 2.25 & 2.29 & 2.28 & 2.32 & 2.31 & 2.32 & 2.39 & 2.49 & 2.77 \\
\hline$R_{\mathrm{m}=0}$ (obs.) & 0.0427 & 0.0439 & 0.0439 & 0.0434 & 0.0438 & 0.0437 & 0.0433 & 0.0544 & 0.0638 \\
\hline$R_{\mathrm{m}= \pm 1}$ (obs.) & 0.0473 & 0.0467 & 0.0466 & 0.0460 & 0.0466 & 0.0467 & 0.0456 & 0.0564 & 0.0643 \\
\hline$R_{\mathrm{m}= \pm 2}$ (obs.) & 0.0521 & 0.0521 & 0.0512 & 0.0511 & 0.0512 & 0.0520 & 0.0500 & 0.0597 & 0.0677 \\
\hline$R_{\mathrm{m}= \pm 3}$ (obs.) & 0.0506 & 0.0518 & 0.0505 & 0.0502 & 0.0503 & 0.0501 & 0.0501 & 0.0595 & 0.0672 \\
\hline$R_{\mathrm{m}= \pm 4}$ (obs.) & 0.0605 & 0.0602 & 0.0602 & 0.0593 & 0.0593 & 0.0605 & 0.0587 & 0.0655 & 0.0740 \\
\hline H-atom treatment & constr. & constr. & constr. & constr. & constr. & constr. & constr. & constr. & constr. \\
\hline Weighting sch. & \multicolumn{9}{|c|}{$\left.w=1 / \sigma^{2}(F)+0.0001 F^{2}\right)$} \\
\hline$\Delta \rho_{\max } /$ e $\AA^{-3}$ & 1.74 & 1.73 & 1.89 & 2.18 & 1.85 & 2.02 & 2.21 & 0.85 & 0.98 \\
\hline$\Delta \rho_{\min } /$ e $\AA^{-3}$ & -1.57 & -1.67 & -1.80 & -1.83 & -1.80 & -1.76 & -1.79 & -0.92 & -0.91 \\
\hline
\end{tabular}

* - pseudomerohedrally twinned with twin volume fractions virtually $0.5: 0.5$, therefore the data are averaged according to Laue class $2 / m$ and the twin fractions are constrained to exactly $0.5: 0.5$

$* *-\alpha=89.9808(13)^{\circ} ; \gamma=89.9763(14)^{\circ}$

$* * *-\alpha=89.9392(2)^{\circ} ; \gamma=89.9939(16)^{\circ}$ 


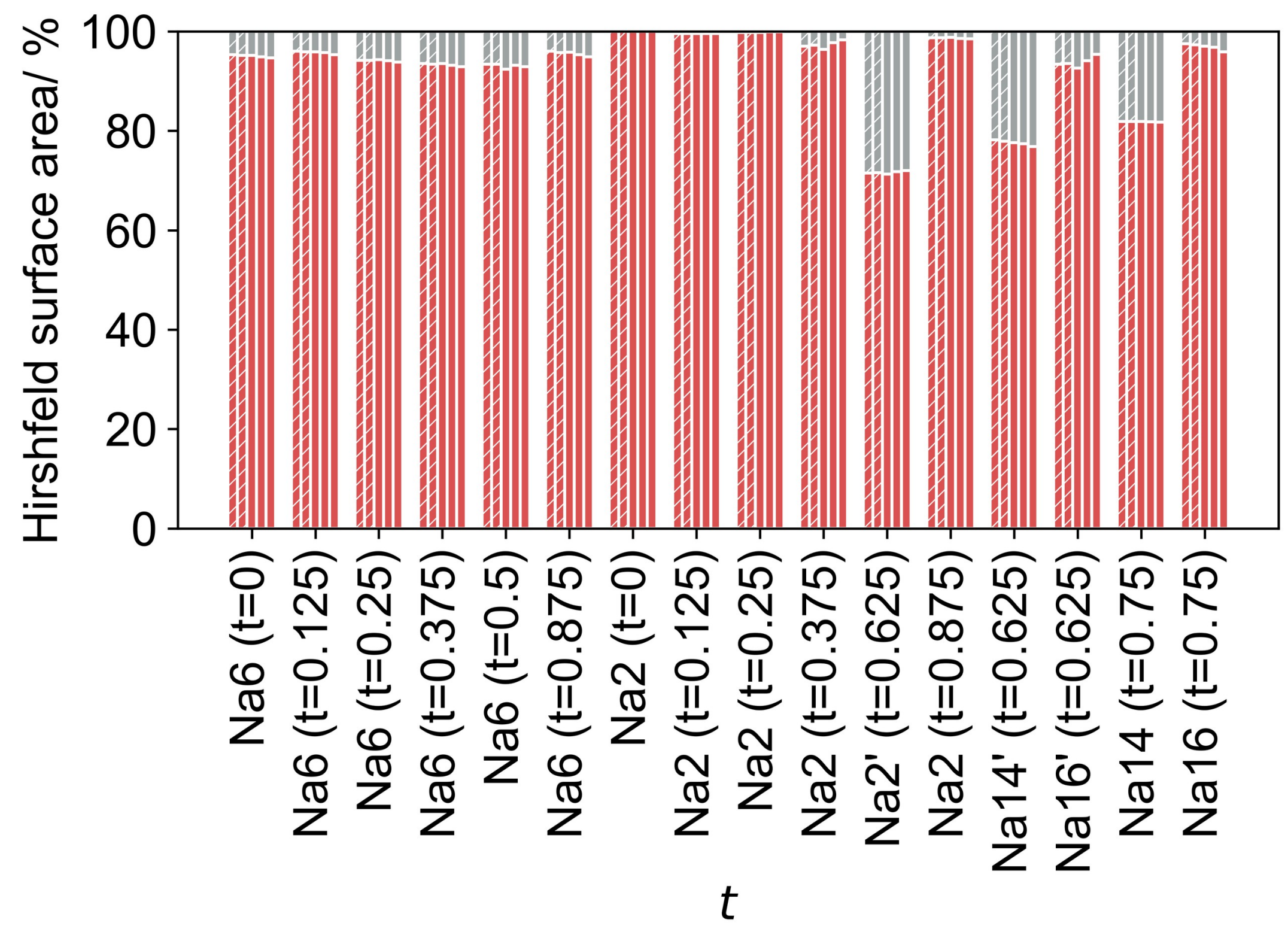

Figure S1. Hirshfeld surface area fractions defined by $\mathrm{Na} \cdots \mathrm{O}$ (red) and Na $\cdots$ other (gray) contacts of cations Na2. The five bars at each $t$ value correspond to structures of primed disorder component at different temperatures (from right to left: 298, 210, 130, 120, and $60 \mathrm{~K}$; phase I - regular, phase II - hatched). 


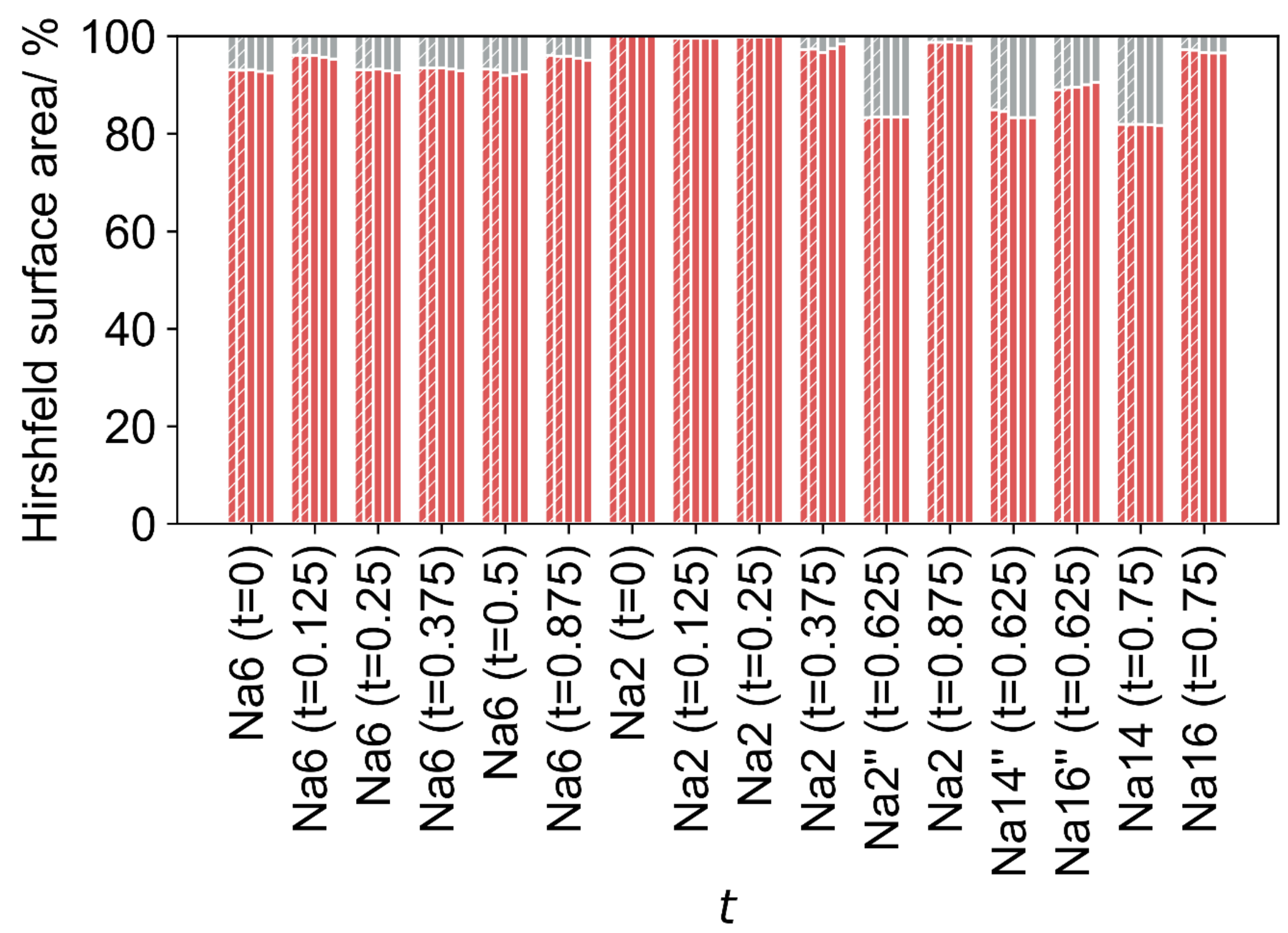

Figure S2. Hirshfeld surface area fractions defined by $\mathrm{Na} \cdots \mathrm{O}$ (red) and $\mathrm{Na} \cdots$ other (gray) contacts of cations $\mathrm{Na}$. The five bars at each $t$ value correspond to structures of double-primed disorder component at different temperatures (from right to left: 298, 210, 130, 120, and $60 \mathrm{~K}$; phase I - regular, phase II - hatched). 